\title{
Adenocarcinoma of the stomach in a tertiary care hospital in Sri Lanka
}

\author{
HDRC Siriwardana' and A Pathirana ${ }^{2}$
}

\begin{abstract}
Objectives Adenocarcinoma of the stomach carries a dismal prognosis when it presents late. Our objective was to describe the location of the tumour, stage at presentation, resectability and survival in a cohort of patients with adenocarcinoma of the stomach, presenting to a tertiary referral centre.
\end{abstract}

Design and setting Data were collected retrospectively from all patients with gastric neoplasms who presented to the University Surgical Unit, Colombo South Teaching Hospital from May 2000 to October 2006.

Results Ninety three patients presented with malignant gastric neoplasms during this period. Majority (86/93) were adenocarcinomas. Mean age at presentation was 58.3 (range 33-83) years. Male to female ratio was 3.15:1. $52.6 \%$ of tumours were in the proximal stomach involving the cardia. Thirty per cent involved the body, and $17.5 \%$ the distal stomach. $93 \%$ had Stage III or IV disease at presentation. Twenty seven patients $(32.5 \%)$ had resectable tumours with a mean survival of 25.3 months. Five year survival was less than $5 \%$, in patients who were not offered surgical resection. There were no patients in our series with early gastric cancer.

Conclusion All our patients presented with advanced gastric cancer and the majority had unresectable disease. The high proportion of patients having proximal gastric carcinoma is similar to the recent changes seen in the west.

\section{Introduction}

Gastric carcinoma was reported to be the fourth commonest cancer worldwide, in the year 2000 [1]. In Sri Lanka, 144 cases were reported in year 2000 [2]. There is little published information on the location of tumour, stage at presentation and resectability, and no data is available on survival.

The objective of this study, conducted at the University Surgical Unit, Colombo South Teaching Hospital, was to document the location, stage at presentation, resectability and survival in a cohort of patients with gastric adenocarcinoma presenting to a tertiary referral centre.

\section{Methods}

The data were collected retrospectively from the unit's oncological database and by direct contact with patients and their families. There were 93 patients with malignant gastric neoplasms who presented from May 2000 to October 2006.

Eighty six $(92 \%)$ of these were adenocarcinomas, $6.4 \%$ $(n=6)$ gastrointestinal stromal tumours and one was a lymphoma. The male to female ratio was $3: 1$. They presented at a mean age of 58 years (range 33-83). Five were below the age of 40 years.

Tumour clearance was considered to be $R_{0}$ when microscopic tumour clearance and lymph node clearance was one level above the involved level, while $\mathrm{R}_{1}$ clearance for microscopically positive margins or when the nodal clearance was same as the level involved.

\section{Results}

\section{Resectability}

Of the 83 patients suitable for surgery, 32 were found to have inoperable tumours on pre-operative investigations (figure 1). The remaining 51, underwent laparotomy but only $27(32.5 \%)$ had resectable tumours. The commonest reason $(84.7 \%$ ) for non-resectability was local infiltration. $\mathrm{R}_{0}$ tumour clearance was achieved in 5 cases while others had $R_{1}$ clearances. The average yield of lymph nodes was 9.2 for each patient with $22 / 27$ cases having positive nodal involvement.

\section{Pathological features}

Of 86 adenocarcinomas $63 \%$ were poorly differentiated and $31 \%$ were moderately differentiated; $39 \%$ were diffuse and $49 \%$ were intestinal according to Lauren's tumour type [3].

Proximal third of the stomach was involved in $52.6 \%$ of the cases, and $29.9 \%$ was in the middle third. The rest involved the distal third of the stomach. The mean maximum diameter of resected tumours was $6.3 \mathrm{~cm}$.

No early gastric cancers were detected, and $93 \%$ were stage III or IV at presentation (table 1).

\footnotetext{
1.2University Surgical Unit, Colombo South Teaching Hospital, Kalubowila, Sri Lanka.

Correspondence: HDRCS, e-mail: <rohansiriwardene@yahoo.com>. Received 12 January, and revised version accepted 29 March 2007. Conflict of interests: none declared.
} 
Table 1. The stage* of gastric adenocarcinoma in our series

\begin{tabular}{cc}
\hline Stage & Percentage $(n=83)$ \\
\hline Ia & - \\
Ib & $2.4 \%(2)$ \\
II & $4.8 \%(4)$ \\
III & $21.4 \%(18)$ \\
IV & $71.4 \%(59)$ \\
\hline
\end{tabular}

* Internationally unified staging for gastric cancer

\section{Survival}

Out of 86 patients survival data was available for analysis in 71 patients (figure 2). The mean survival in advanced unresectable patients $(\mathrm{n}=56)$ was 5 months (range 1-24), and 5-year survival was zero. A mean survival of 25.3 months (5-60 months) and five-year survival of $13.8 \%$ was seen following gastric resection. The mean survival with positive and negative margins was 18.2 and 27.3 months respectively. In the resected group of patients $(\mathrm{n}=27)$, in-hospital mortality was $7.4 \%(2 / 27)$.

\section{Discussion}

In our series, the mean age at presentation was 58 years. According to the Sri Lanka cancer registry and other available data, the mean age at presentation of patients with gastric cancer is between 50 and 55 years $[2,4]$. In most western data the age at presentation is about one decade higher than ours $[5,6]$.

In 1984 at Nawalapitiya Base Hospital Dissanayake and Colombage reported that $74 \%$ of the tumours to involve the antrum of the stomach [4]. International data describe the change in the location of tumour from the antrum to cardia. Some series document that more than $50 \%$ of the tumours involve the proximal stomach $[7,8,9]$. In our series $52 \%$ of the tumours were found in the proximal third of the stomach.

Figure 1. The summary of management protocol and the interventions done

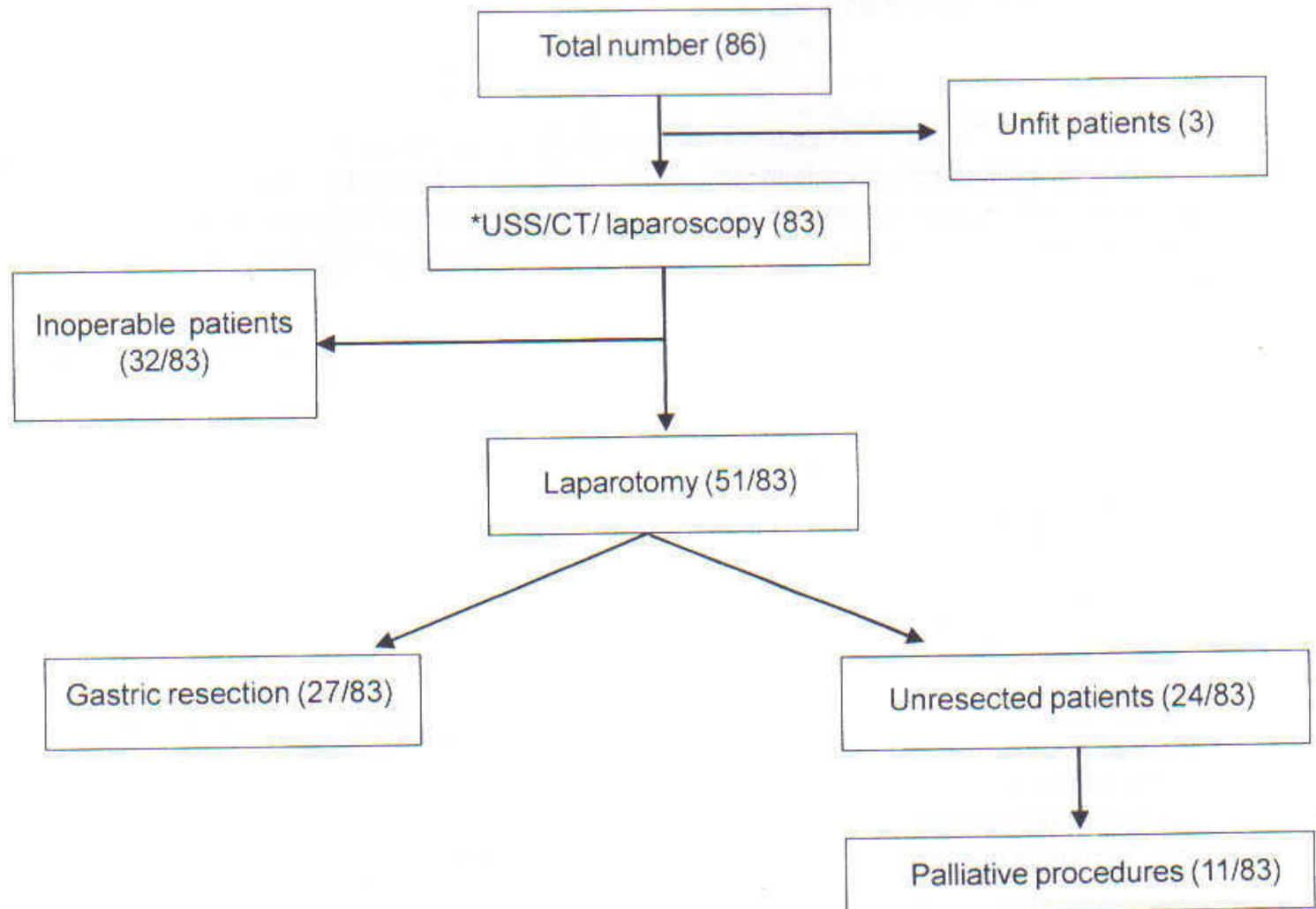

("USS/CT ultrasound/CT scan of abdomen) 
Table 2. Comparison of results with 18365 USA patients [6]

\begin{tabular}{lcccccc}
\hline & Stage I & Stage II & Stage III & Stage IV & Resectability & Overall 5 year survival \\
\hline $\begin{array}{l}\text { Our series } \\
(\mathrm{n}=86)\end{array}$ & $2.4 \%$ & $4.8 \%$ & $21.4 \%$ & $71.4 \%$ & $32.5 \%$ & $4.8 \%$ \\
$\begin{array}{l}\text { Wanebo et.al. } \\
(\mathrm{n}=18365)[6]\end{array}$ & $17 \%$ & $17 \%$ & $35 \%$ & $31 \%$ & $70 \%$ & $14 \%$ \\
\hline
\end{tabular}

Kaplan-Meier survival curve for Gastric cancer

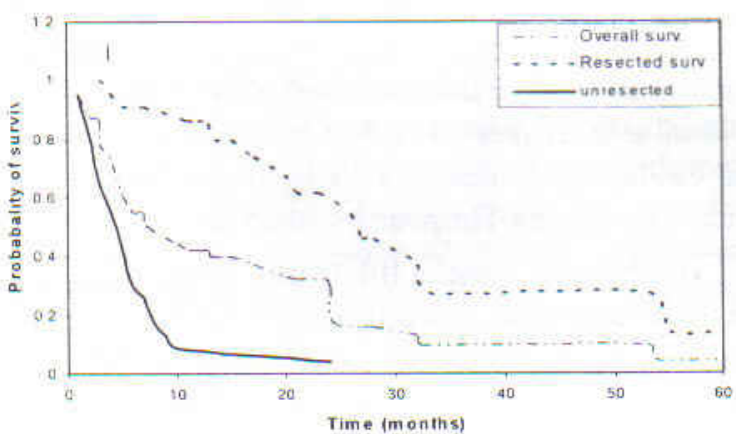

Figure 2. 5-year survival.

Seventy two percent of our series were stage IV at presentation and overall resectability was $32.5 \%$. The overall 5 -year survival is less than $5 \%$ and in resected patients the 5 -year survival was $13.8 \%$. The stage specific survival was not calculated because of the small sample size. In comparison, a large USA study [6] reported only $31 \%$ to be at stage IV at the presentation with a $70 \%$ overall resectability. Their overall 5 -year survival was $14 \%$ and survival following resection was $19 \%[6]$.

\section{Conclusions}

A majority of the tumours in our series involving the proximal third of the stomach is similar to the trends in other series. The patients presented at an advanced unresectable stage that resulted in a low overall 5-year survival rate $(<5 \%)$. Awareness of gastric cancer needs to be increased in Sri Lanka, and studies should be carried out to identify the population at risk.

\section{References}

1. Parkin DM. Global cancer statistics in the year 2000 . Lancet Oncology 2001; 2: 533-43.

2. Sri Lanka Cancer Registry - National Cancer Control Program 2000.

3. Laurén P. The two main histological types of gastric carcinoma: diffuse and so-called intestinal-type carcinoma. Acta Pathologica et Microbiologica Scandinavica $1965 ; 64:$ 31-49.

4. Dissanayake AL, Colombage GR. The pattern of gastric cancer in a district hospital in Sri Lanka. Journal of Royal College of Surgeons Edinburgh 1984; 29: 22-6.

5. Ferlay J, Bray F, Pisani P. GLOBOCAN 2002: Cancer incidence, mortality and prevalence worldwide. Version 2.0. Lyon: IARC Press, 2004. (http://www-depdb.iarc.ff/ globocan2002.htm)

6. Wanebo HJ, Kennedy BK, Chmiel J, Steele G, Winchester D, Osteen R. Cancer of the stomach. A patient care study by the American College of Surgeons. Annals of Surgery 1993; 218: 583-92.

7. Allum WH, Powell DJ, McConkey CC, Fielding JWL. Gastric cancer: a 25 year review. British Journal of Surgery 1989; 76: 535-40.

8. Paterson IM, Easton DF, Corbishley CM. Changing distribution of the adenocarcinoma of the stomach. British Journal of Surgery 1987; 74: 481-2.

9. Devesa SS, Blot WJ, Fraumeni JF, Changing patterns in the incidence of esophageal and gastric carcinoma in the United States. Cancer 1998; 83: 2049-53. 